Revue internationale P.M.E.

Économie et gestion de la petite et moyenne entreprise

Revure

internationale

PME

\title{
Interaction entre l'environnement universitaire et les premières années des entreprises essaimantes canadiennes
}

\section{Jérôme Doutriaux}

Volume 5, numéro 2, 1992

URI : https://id.erudit.org/iderudit/1008137ar

DOI : https://doi.org/10.7202/1008137ar

Aller au sommaire du numéro

Éditeur(s)

Presses de l’Université du Québec

ISSN

0776-5436 (imprimé)

1918-9699 (numérique)

Découvrir la revue

Citer cet article

Doutriaux, J. (1992). Interaction entre l'environnement universitaire et les premières années des entreprises essaimantes canadiennes. Revue internationale P.M.E., 5(2), 7-39. https://doi.org/10.7202/1008137ar

\section{Résumé de l'article}

ette étude du développement d'entreprises à base technologique issues d'universités canadiennes confirme l'importance du climat universitaire, des conditions de démarrage, et particulièrement des caractéristiques personnelles des entrepreneurs. Les entreprises qui se développent le mieux ont en effet tendance à venir d'universités ayant une bonne infrastructure de support à la recherche et des liaisons avec l'industrie, à avoir une orientation technologique et à être dirigées par un entrepreneur ayant quitté le monde académique. L'analyse, faisant appel à une série d'analyses factorielles, est basée sur l'observation de 58 entreprises créées entre 1971 et 1990 par des universitaires canadiens en science, science appliquée et génie pour exploiter commercialement des inventions issues de leurs laboratoires. 


\title{
Interaction entre l'environnement universitaire et les premières années des entreprises essaimantes canadiennes
}

Jérôme DOUTRIAUX*

Université d'Ottawa

\begin{abstract}
RÉSUMÉ
Cette étude du développement d'entreprises à base technologique issues d'universités canadiennes confirme l'importance du climat universitaire, des conditions de démarrage, et particulièrement des caractéristiques personnelles des entrepreneurs. Les entreprises qui se développent le mieux ont en effet tendance à venir d'universités ayant une bonne infrastructure de support à la recherche et des liaisons avec l'industrie, à avoir une orientation technologique et à être dirigées par un entrepreneur ayant quitté le monde académique. L'analyse, faisant appel à une série d'analyses factorielles, est basée sur l'observation de 58 entreprises créées entre 1971 et 1990 par des universitaires canadiens en science, science appliquée et génie pour exploiter commercialement des inventions issues de leurs laboratoires.
\end{abstract}

\section{ABSTRACT}

This analysis of the development of university technology-based spin-off firms confirms the importance of university climate, of start-up conditions, and, even more, of the personal characteristics of the entrepreneurs. Indeed, most

* Jérôme Doutriaux est professeur titulaire à la Faculté d'administration de l'Université d'Ottawa et directeur associé de son programme de maîtrise de gestion en ingénierie. Après des études d'ingénieur en France (ICAM, ESE), il a obtenu un doctorat en science des systèmes aux États-Unis (Université Carnegie-Mellon). Il est coauteur de plusieurs livres en économie d'entreprise. Ses articles sur la création de petites entreprises à technologie avancée et le transfert de technologie ont paru dans diverses revues scientifiques telles que Journal of Business Venturing, IEEE Transactions on Engineering Management, Journal of Small Business and Entrepreneurship et dans plusieurs livres techniques. Adresse : Faculté d'Administration, Université d'Ottawa, Ottawa K1N 6N5.

Cette recherche a été financée en partie par le Conseil de recherche en sciences humaines. Les conclusions sont celles de l'auteur et ne correspondent pas nécessairement aux positions de l'agence de financement. 
successful firms tend to come from universities with a good infrastructure supporting research and industry linkages, to be technology oriented at startup, and to be directed by entrepreneurs who have left their university. The study, using multivariate analyses, is based on the observation of 58 entrepreneurial firms created between 1971 and 1990 by members of science, applied science and engineering Faculties at Canadian Universities to further develop and exploit commercially inventions originating in their laboratories.

\section{RESUMEN}

Este análisis del desarrollo de las empresas de base tecnológica generadas por las univerdidades confirma la importancia que tienen el ambiente universitario, las condiciones de inicio y especialmente las características personales de los innovadores. La mayoría de las empresas con éxito tienden a ser generadas por universidades que cuentan con una infraestructura adecuada fomentado la investigación y los vínculos con la industria, además estas empresas tienen una orientación tecnológica al inicio, y son dirigidas por innovadores quienen abandonaron su universidad. El estudio, usando análisis de variables múltiples, está basado en la observación de 58 firmas innovadoras creadas entre 1971 y 1990 por miembros de las facultades de ciencias, ciencias aplicadas $\theta$ ingenieria de universidades Canadienses para continuar con el desarrollo y explotar comercialmente inventos originados en sus laboratorios.

\section{Introduction}

Alors qu'il recevait peu de soutien au cours des années 70 , l'essaimage universitaire est maintenant toléré et même soutenu par beaucoup d'universités pour son potentiel de transfert de technologie vers l'industrie. Les conditions qui favorisent le bon démarrage et la croissance des entreprises créées par un membre d'une communauté universitaire pour développer et commercialiser une invention ne sont encore que partiellement connues. Les chercheurs ont en effet montré que ces entreprises diffèrent des autres entreprises à base technologique à cause de l'environnement universitaire dont elles sont issues et du statut spécial de leurs fondateurs qui ont souvent tendance à combiner leurs activités professorales et entrepreneuriales.

Cette étude présente une analyse empirique des facteurs qui influencent le développement initial d'entreprises créées par des universitaires canadiens pour exploiter commercialement des idées ou inventions issues des laboratoires universitaires. Elle est basée sur l'hypothèse selon laquelle l'environnement et la culture universitaire affectent les conditions de démarrage des entreprises et que le succès de ces entreprises résulte partiellement de la combinaison de cette culture et de ces conditions de démarrage. La culture universitaire est mesurée 
selon la charge d'enseignement et l'intensité de recherche, les règles de promotion et de permanence, l'infrastructure favorisant les contacts avec l'industrie et l'entrepreneuriat. En exposant les conditions du succès des entreprises essaimantes, cette analyse devrait être utile aux administrateurs du transfert de technologie des universités, aux entrepreneurs potentiels et aux planificateurs gouvernementaux.

L'analyse est basée sur l'observation des conditions de démarrage et des premières années de 58 entreprises créés au Canada entre 1971 et 1990 par des membres de facultés de science, génie, et sciences appliquées, alors qu'ils étaient encore à l'université ou juste après leur départ, pour exploiter commercialement des idées ou inventions issues des laboratoires universitaires.

Le modèle conceptuel sur lequel est basé cette étude suit une brève revue de la littérature ; l'échantillon utilisé est ensuite décrit. L'analyse qui suit, basée sur une série d'analyses factorielles et de corrélations, confirme l'importance du climat universitaire et de la motivation de l'entrepreneur : les entreprises qui se développent le mieux ont tendance à venir d'universités ayant un forte orientation vers la recherche et une infrastructure favorisant les échanges avec l'industrie et à être dirigées par un entrepreneur ayant quitté le monde universitaire.

\section{Analyse de la littérature et cadre conceptuel}

Au cours des dix dernières années, l'attitude des universités sur la collaboration avec l'industrie a changé considérablement. Alors que les programmes de recherche avec une tournure industrielle trop prononcée ou la formation professionnelle "sur mesure » étaient découragés ou regardés avec suspicion pour leurs atteintes à la " pureté » de la recherche fondamentale et de l'offre d'une formation universitaire de base (Doutriaux et Peterman, 1982), bon nombre d'universités développent maintenant activement leurs liens avec l'industrie (Conseil des sciences du Canada, 1988): formation professionnelle continue, séminaires, projets de recherche conjoints, recherche sous contrat, échange de personnel scientifique, exploitation conjointe de brevets d'invention, création d'entreprises. Le resserrement des liens université-industrie a plusieurs avantages : augmentation de la visibilité des universités en tant qu'agent de changement social et industriel par la formation appliquée, augmentation des possibilités de recherche ou des fonds disponibles pour les chercheurs et réorientation de la recherche vers la solution de problèmes industriels immédiats.

La commercialisation de la recherche universitaire, plus pour son effet d'entraînement sur les activités de recherche que pour les revenus espérés qui 
sont, en réalité, assez limités, constitue l'un des aspects de la coopération université-industrie les plus intéressants pour les administrateurs universitaires et les chercheurs (Munsche, 1990). Cette commercialisation peut prendre plusieurs formes : vente ou licence de brevet ou création d'une filiale commune avec une entreprise existante ou création par l'inventeur d'une nouvelle entreprise indépendante. Dans tous les cas, les différences entre les cultures industrielles et universitaires sont sources de conflits : échange libre de résultats de recherche ou secret industriel, priorité donnée à la recherche fondamentale à long terme ou à la solution de problèmes immédiats avec retour financier rapide (Rogers, 1986 ; Dill, 1990). Chaque méthode de commercialisation a ses avantages et ses inconvénients. Les licences ont tendance à soutenir les inventions intéressantes à court terme, à séparer l'inventeur de l'application de son invention et donc à réduire les chances d'amélioration ou de nouveaux développements, à mener à des conflits lors de la distribution des royautés, à ne pas forcément avoir de retombées économiques dans la région de l'université (Brown, 1984). La création par l'inventeur d'une entreprise pour exploiter indépendamment son invention occasionne également des problèmes : l'inventeur universitaire n'a pas toujours le temps, la motivation, les compétences requises pour gérer une entreprise. De plus, le temps passé dans l'entreprise peut réduire son efficacité de chercheur (McMulan et Melnyck, 1988). L'entrepreneur universitaire doit aussi être capable de concilier la dynamique, les objectifs et les exigences souvent divergents de la vie scolaire et du monde des affaires, ce qui est une difficulté supplémentaire (Van Dierdonck et Debackere, 1988).

L'effet direct de la culture et des politiques universitaires sur les entreprises essaimantes n'est pas très clair et varie dune institution à l'autre comme le montrent les études de cas publiées sur l'entrepreneuriat technologique et la gestion de la technologie. L'évolution de l'attitude des universités envers l'entrepreneuriat universitaire, hostile il y a encore quelques années et maintenant tolérante et même favorable, rend difficile toute analyse systématique. En Suède, Olofsson et Wahlbin (1984) ont montré qu'à la fin des années 70 et au début des années 80, les entreprises essaimantes avec financement externe et indépendantes des universités réussissaient mieux que les autres. Des résultats similaires ont été obtenus au Canada (Doutriaux, 1987), confirmant des observations antérieures (Doutriaux et Peterman, 1982). Une étude suédoise plus récente semble montrer que le changement des attitudes universitaires et de l'environnement (capital de risque) a mené, à la fin des années 1980, à une augmentation de l'essaimage universitaire et à un taux de croissance plus élevé des entreprises (Olofsson et alii, 1987). Dans une étude de l'essaimage universitaire autour de Cambridge University, Segal (1986) a remarqué que la création d'entreprises s'est faite sans intervention ou support particulier de l'université, l'accent mis par l'université sur l'excellence de l'enseignement et de la recherche étant 
probablement la raison la plus probante du développement d'excellentes relations avec l'industrie. Ce résultat est confirmé par une autre étude récente d'entrepreneurs scientifiques qui a avancé l'hypothèse que l'activité entrepreneuriale est due plus à la dynamique de groupe locale qu'aux politiques et structures universitaires (Louis et alii, 1989). L'expérience de Bagby et Rao (1990) montre tout de même que le support de l'université (bourses de recherche et de démarrage surtout), lorsque disponible, est utilisé efficacement par les entrepreneurs universitaires, en particulier au cours des premières années de leurs entreprises.

Selon ce court survol de la littérature, la culture universitaire, l'environnement et les conditions de départ s'avèrent d'importants déterminants du succès des entreprises à base technologique issues des universités. C'est pourquoi ces trois types d'effets sont utilisés comme cadre conceptuel pour cette recherche. Les facteurs considérés pour leur impact potentiel sur les entreprises émergentes sont résumés à la figure 1 ; la majorité provient de notre brève analyse de la littérature, tandis que les autres ont été suggérés au cours d'entrevues avec des entrepreneurs.

Dans cette étude, 84 variables sont utilisées pour représenter divers aspects des éléments du cadre conceptuel de la figure 1 . Ces variables ont été sélectionnées à la suite d'entrevues avec des doyens, des responsables de bureaux de

FIGURE 1

\section{Cadre conceptuel : facteurs affectant le développement de l'entreprise}

\begin{tabular}{|l|l|}
\hline $\begin{array}{l}\text { Culture Universitaire } \\
\text { - Orientation vers la recherche } \\
\text { - Politiques d'enseignement, } \\
\text { technologie, promotions } \\
\text { - Infrastructure } \\
\text { - Esprit d'entreprise } \\
\text { - Liens avec l'extérieur } \\
\text { - Ressources }\end{array}$ \\
\begin{tabular}{|l|l|}
\hline $\begin{array}{l}\text { L'environnement } \\
\text { - Orientation régionale } \\
\text { vers la technologie } \\
\text { - Ressources locales } \\
\text { - Programmes de support }\end{array}$ \\
\hline
\end{tabular} \mid $\begin{array}{l}\text { Conditions économiques } \\
\text { Orientation « recherche » } \\
\text { Expérience } \\
\text { - Organcement } \\
\text { Orientation initiale }\end{array}$ \\
\hline
\end{tabular}


transfert de technologie et des entrepreneurs en fonction de ce qui était disponible ou possible d'obtenir dans la majorité des universités et entreprises. Certaines variables, en particulier celles qui sont associées aux conditions de démarrage et au développement des entreprises, ainsi qu'aux caractéristiques managériales des entrepreneurs, ont déjà été utilisées pour l'analyse d'entreprises émergentes à haute technologie (voir par exemple Doutriaux, 1992). Les autres variables, reliées à la culture universitaire et à l'environnement, sont plus inédites dans ce contexte, la nature plus descriptive des études antérieures sur l'essaimage universitaire n'ayant pas requis le développement de mesures quantitatives.

La liste détaillée des variables, leur description et leur échelle de mesure sont données en annexe. En accord avec le cadre conceptuel de l'étude, ces variables sont liées aux concepts suivants :

\subsection{Culture universitaire et environnement (35 varlables)}

- liaisons avec l'extérieur, comité consultatif, programmes coopératifs, participation à des réseaux de recherche externes, existence d'un bureau de liaison industrielle ;

- taille, importance et source des fonds de recherche, charge d'enseignement ;

- politiques de promotion et de permanence ;

- activités du bureau de liaison industrielle ;

- orientation urbaine, industrielle et linguistique de la région.

\subsection{Conditions de démarrage (36 variables)}

- position et expérience universitaire et industrielle de l'entrepreneur universitaire ;

- objectifs de l'entrepreneur au moment du démarrage (sa carrière, son entreprise) ;

- expertise managériale de l'équipe de fondateurs au moment du démarrage ;

- capital initial et sources des fonds ;

- similitude entre la technologie utilisée par l'entreprise et celle du laboratoire universitaire ;

- productivité en recherche universitaire du fondateur principal avant le démarrage ;

- aide administrative reçue de l'université au moment du démarrage. 


\subsection{Développement de l'entreprise et activités de l'entrepreneur ("Indicateurs de performance ", 13 variables)}

- niveau des ventes au cours des cinq premières années ;

- structure financière actuelle, niveau de contrôle conservé par l'entrepreneur ;

- productivité du fondateur principal en recherche universitaire après le démarrage ;

- position actuelle de l'entrepreneur à l'université et temps passé dans l'entreprise ;

- évaluation subjective par l'entrepreneur de l'état de son entreprise.

Certaines mesures, reliées à la « productivité créatrice » des universités (nombre d'inventions, nombre de brevets), à certaines caractéristiques des activités de recherche (sources détaillées de fonds, nombre de contrats), à certains critères de promotion et de permanence, à des conditions de départ, n'ont pas été utilisées à cause d'un trop grand nombre d'observations manquantes ou de difficultés à effectuer des mesures homogènes dans l'ensemble des universités.

Cette étude préliminaire, assez descriptive, s'intéresse à l'impact général de la culture universitaire sur le processus de création d'entreprise et sur le développement initial de ces entreprises, ainsi qu'à l'effet spécifique de certaines orientations et politiques universitaires. Les propositions de recherche pertinentes sont les suivantes :

1) Quelles sont les caractéristiques de la culture universitaire et de l'environnement qui affectent le plus les conditions de démarrage des entreprises?

2) Quel est l'effet à court terme sur l'entreprise et sur l'entrepreneur de l'environnement universitaire et des conditions de démarrage de l'entreprise ?

3) Y a-t-il incompatibilité entre productivité en recherche et succès comme entrepreneur?

4) Le bureau de transfert de technologie (BTT) des universités a-t-il un effet positif sur le développement des entreprises essaimantes ?

En fonction des conclusions des études décrites dans la littérature, on peut s'attendre à observer une interaction significative entre la culture universitaire et les conditions de démarrage, ainsi qu'un effet significatif sur le développement de l'entreprise issue d'un environnement universitaire et sur ses conditions 
de démarrage. Par contre, à cause des différences de dynamique entre le monde des affaires et celui de la recherche, on s'attendrait à observer une incompatibilité entre productivité en recherche et succès entrepreneurial. De plus, l'effet du BTT sur les entreprises est difficile à prévoir : il existerait un effet positif potentiel, car son existence indique l'intérêt de l'université pour les contacts industriels, et un effet négatif potentiel, car un BTT pourrait essayer d'appliquer les valeurs et la dynamique universitaire au monde des affaires.

\section{Collecte des données et caractéristiques de l'échantillon}

Cette étude est basée sur une enquête menée auprès des bureaux de liaison industriels, des doyens, et d'entrepreneurs œuvrant dans des universités canadiennes. Trois types de questionnaires ont été envoyés aux institutions membres de l'Association des universités et collèges du Canada (AUCC, 1989) ayant des facultés d'agriculture, génie, médecine, santé, sciences et sciences appliquées : un pour le bureau de transfert de technologie («BTT »), un pour chaque doyen («DOYENS ») et un pour les entrepreneurs universitaires (« ENTREP ») à être distribué par le BTT, les doyens et les entrepreneurs rejoints à tout entrepreneur connu ou probable. Des questionnaires ont aussi été distribués directement à des représentants de BTT canadiens en juillet 1990 lors de la réunion annuelle de l'Association of University Technology Managers à Vancouver, Canada. On a aussi demandé à chaque doyen de distribuer à tous ses professeurs une note qui décrit ce projet et qui encourage les professeurs à demander une copie du questionnaire. Trouver les entrepreneurs universitaires est un travail de détective compliqué par des questions de confidentialité : peu d'universités ont des listes de leurs entreprises essaimantes; et il n'y a pas de consensus sur la définition d'une telle entreprise. Certaines universités n'incluent sur leurs listes que les entreprises crées avec leur aide pour exploiter un brevet universitaire ; d'autres considèrent que toute entreprise créée par un ancien élève ou par un membre du corps professoral est une entreprise essaimante, même si elle ne fait pas appel à une technologie issue de l'université. Pour notre étude, une entreprise essaimante doit avoir été créée par un universitaire (professeur, chercheur, étudiant), seul ou avec l'aide de l'université, en vue de l'exploitation commerciale d'une idée ou d'une invention universitaire.

Cinquante doyens (sur 112) de 31 universités (sur 53) ont retourné leur questionnaire ; quatre ont décidé de ne pas participer à l'étude, jugeant que les activités de recherche dans leurs facultés étaient trop faibles. Des réponses de 35 universités $(66 \%)$ furent reçues des bureaux de transfert de technologie (ou 
d'administration de la recherche), six répondants d'universités peu actives en recherche renvoyant des questionnaires non remplis. Sur un total de 96 questionnaires complétés par des entrepreneurs, 91 étaient utilisables. La représentativité de cet échantillon de 91 entreprises est difficile à estimer, car le nombre réel d'entreprises d'origine universitaire au Canada est inconnu. En 1986, Philip Enros du Conseil des sciences du Canada (1987) a évalué qu'il y avait environ 125 entreprises de ce genre dans le pays. En 1990, le nombre a probablement augmenté, mais ni les doyens, ni les bureaux de transfert de technologie n'ont de statistiques précises.

Cette analyse est basée uniquement sur les 58 entreprises créées entre 1971 et 1990 par des professeurs de science (31) ou de science appliquée et de génie (27). Une majorité d'entrepreneurs étaient professeurs titulaires au moment de la création de leur entreprise (39), un bon nombre étant professeurs agrégés (15), un étant professeur adjoint et trois étant chercheurs ou étudiants. Presque tous avaient des postes permanents (98\%). Les entreprises viennent de 21 universités (une université avec sept entreprises essaimantes, deux universités avec cinq entreprises, une université avec quatre entreprises, les autres avec trois ou moins de trois entreprises dans l'échantillon). Environ $20 \%$ des répondants sont francophones. Les données recueillies auprès des doyens et des bureaux de transfert de technologie ont été appariées avec les données des entrepreneurs. Les variables retenues pour cette étude sont données en annexe. Celles qui sont reliées aux conditions de démarrage des entreprises ont généralement plus de 54 observations utiles, à l'exception des niveaux annuels de ventes qui ont de 43 (premières années) à 33 observations (cinquième année). Les variables reliées à l'environnement universitaire ont de 41 à 53 observations utiles.

L'échantillon contient des entreprises entrepreneuriales assez typiques : en dollars constants de 1986, les ventes moyennes de la première année sont de $85744 \$$ (de zéro à plus de 500000 \$); les ventes moyennes de la cinquième année sont de 826250 \$ (de 1000 \$à plus de 10000000 \$). L'emploi moyen à temps plein au cours de la première année est de 2,2 personnes, et de 8,6 au cours de la cinquième année. Les revenus de première année ( $20 \%$ des produits, $60 \%$ des services et $20 \%$ d'autres revenus) ont été assez bien distribués entre le gouvernement (39\%, ventes militaires non comprises) et l'industrie (35\%), le reste étant moins important (ventes aux consommateurs, ventes militaires, etc.). Le financement de départ provenait surtout de l'entrepreneur, de sa famille et de ses amis (65\% en moyenne), de bourses gouvernementales (13\%), prêts bancaires $(11 \%)$, capital risque $(7 \%)$, la part des universités étant très faible (moins de $2 \%$ en moyenne, zéro le plus souvent).

Environ $59 \%$ du capital initial (valeur moyenne de $112000 \$$ (dollars de 1986) est venu des fondateurs ou de leurs partenaires qui contrôlaient $92 \%$ de 
l'entreprise au début ; seulement deux entreprises ont reçu du financement de leur université au départ, l'une $20 \%$ et l'autre $100 \%$ du capital initial, et seulement deux étaient contrôlées par l'université (respectivement $6 \%$ et $50 \%$ du capital-action).

Le nombre moyen de fondateurs était 2,7 (de 1 à 7 personnes), $77 \%$ provenant de l'université. Seulement $46 \%$ des entrepreneurs qui ont complété un questionnaire avait travaillé à temps plein dans l'industrie (pour 5,9 ans en moyenne) avant de créer leur entreprise. Ils avaient passé en moyenne 15,7 ans dans leur université avant de le faire. Au cours de la première année, une moyenne de seulement $5 \%$ de l'administration de l'entreprise était assurée par des gestionnaires professionnels ; au bout de cinq ans, ce pourcentage était passé à $14 \%$. Les entrepreneurs considèrent qu'ils avaient un style de gestion assez équilibré : score moyen de 5,6 sur une échelle continue allant de 0 (style autoritaire et directif) à 10 (pleine participation de tous). Plusieurs entreprises ont commencé avec un plan d'affaire (58\%) et avec un plan marketing (32\%). Les entrepreneurs considèrent qu'au début leurs entreprises avaient plutôt une orientation technologique (score de 3,2 sur une échelle de 0 (technologie) à 10 (marketing)). Environ $26 \%$ des entreprises exploitent des brevets qui, dans $35 \%$ des cas, appartiennent à l'université. L'expérience passée des fondateurs était principalement en $R$ et $D$, avec des compétences en administration et en finance, mais ils avaient peu d'expertise en marketing et en passation de contrats avec le gouvernement ; $34 \%$ des entrepreneurs avaient déjà de l'expérience en démarrage d'entreprise.

Les statistiques qui suivent sont pondérées en fonction du nombre d'entrepreneurs issus des universités; elles ne sont donc pas forcément représentatives de l'ensemble des universités canadiennes. En moyenne, la charge d'enseignement dans les facultés d'où viennent les entrepreneurs est de 3,9 cours par an, $85 \%$ de l'enseignement étant assuré par le corps professoral à temps plein (63\% à $96 \%$ ). Le budget de recherche moyen par professeur est de 66000 \$ venant pour $57 \%$ des grandes agences canadiennes de recherche (CRSNG, CRM, $\mathrm{CRSH}$ ), pour $14 \%$ de l'industrie et pour $29 \%$ d'autres sources (services publics, organismes internationaux, fondations, budget universitaire). Les entrepreneurs de l'échantillon viennent d'universités ayant presque toutes un bureau de transfert de technologie ou de liaison université-industrie (créé il y a 6 ans en moyenne) ; $40 \%$ des entrepreneurs viennent d'universités avec un centre d'innovation. Pour ce qui est de l'évaluation des dossiers des professeurs en vue de la permanence et des promotions, le poids relatif moyen donné aux publications est de $52 \%$ contre $35 \%$ à l'enseignement et $13 \%$ aux activités administratives et de service à la communauté. Enfin ,95\% des entrepreneurs viennent d'universités où il est possible d'obtenir un poste à temps partiel permettant de passer plus de temps dans une entreprise ou dans d'autres activités externes. 


\section{Résultats}

Comme nous l'avons mentionné, dans une section précédente, l'objectif de cette étude est d'analyser l'impact de la culture universitaire et environnementale ainsi que des conditions de démarrage sur le développement des entreprises essaimantes. La culture et les conditions de départ étant représentées par un assez grand nombre de variables mesurant des caractéristiques quelquefois indépendantes et quelquefois similaires, des analyses factorielles sont utilisées pour identifier leur structure. Les facteurs décrivant cette structure sont utilisés pour construire des modèles expliquant certaines caractéristiques de développement des entreprises dans l'échantillon.

Ces analyses factorielles sont basées sur les 35 variables « culture et environnement » et sur les 36 variables " conditions de démarrage » décrites dans une section précédente. Dans les deux analyses, les données manquantes ont été remplacées par la moyenne des observations de façon à permettre une analyse utilisant le plus grand nombre de cas possible.

\subsection{Les dimensions de la " culture universitaire et de l'environnement "}

Les 35 variables « culture et environnement » présentées au tableau 1 peuvent être résumées par 10 «facteurs universitaires » (FU1 à FU10) qui, ensemble, expliquent $84 \%$ de la variance des variables originales, les autres facteurs potentiels ne contribuant que marginalement (moins que la moyenne) à la variance ayant été ignorés.

Les dimensions de la culture universitaire peuvent être inférées à partir des corrélations ou des « facteurs de pondération » du tableau 1. Les composantes de chacune de ces dimensions sont notées ci-dessous :

FU1 : BTT, orientation « recherche » et liens de recherche avec l'extérieur

- présence d'un bureau de transfert de technologie dans l'université ;

- importance des publications dans l'évaluation des professeurs en vue d'une promotion ;

- participation active de la faculté à des instituts de recherche et à des centres d'excellence ;

- effet négatif (faible) des activités externes non directement universitaires des professeurs (ACTEXT) lors des évaluations pour les promotions et la permanence. 
TABLEAU 1

Analyse factorielle, culture universitaire et environnement corrélations $(\leq .30)$ des variables et des dimensions

\begin{tabular}{|c|c|c|c|c|c|c|c|c|c|c|}
\hline $\begin{array}{l}\text { Facteur } \\
\text { Variable }\end{array}$ & FU1 & FU2 & FU3 & FU4 & FU5 & FU6 & FU7 & FU8 & FU9 & FU10 \\
\hline INST & 69 & .31 &. .30 & & & & & & & \\
\hline RESEAU & .93 & & & & & & & & & \\
\hline concons & & & & .60 & & & .30 & & .30 &. .42 \\
\hline PUBL & .46 & & &. .41 & .30 & .63 & & & & \\
\hline ACTEXT & -.72 & & & .35 &. .40 & & & & .36 & \\
\hline EVALENS & .34 & & & & .71 & & & & & \\
\hline CRSENIR & & & -.82 & & & & & & & \\
\hline$\infty$ & & & & & & & .69 & & .33 & \\
\hline BTT & .93 & & & & & & & & & \\
\hline INNOV & .30 & -.31 & -.35 & 48 & -.30 & & & & .32 & .33 \\
\hline ACCESLAB & & & & & & & & & .94 & \\
\hline BUDRPROF & & .38 & & & .74 & & & & & \\
\hline cHARGENS & &. .30 & & & & & -.51 & & & -.41 \\
\hline ENSTPL & & & & -.79 & & & & & & \\
\hline PHDSPR & .38 & .53 & & & & & & & & \\
\hline PHDS & & .92 & & & & & & & & \\
\hline NTPFAC89 & & .86 & & & & & & & & \\
\hline OCTROISR & & &. .37 & & -.67 & & & .46 & & \\
\hline INDUST & & & .37 & .71 & & & & & & \\
\hline TPARTIEL & & & & & & & & & & .87 \\
\hline SUPPORTF & .36 & & .52 & & & & & & & \\
\hline LANGUAGE & & & & .86 & & & & & & \\
\hline URBAIN & & .30 & .78 & & & & & & & \\
\hline HCHIECH & & .30 & .78 & & & & & & & \\
\hline EMPBTT & & .30 & & & & & & .57 & .40 & .34 \\
\hline CONBTT & &. .32 & & & & & & .80 & & \\
\hline BTTINIT & & -.31 &. .54 &. .32 &. .30 & & & .38 & & \\
\hline INVRISO & & & .37 &. .38 &. .36 & & &. .41 & & \\
\hline AGEBTT & & & & & .72 & & & & & \\
\hline PROACT & & & & & & .96 & & & & \\
\hline REACT & & & & & &. .97 & & & & \\
\hline BUDRUNI & & & & & & & .84 & & & \\
\hline CRSNGSH & & & & & .48 & &. .47 & & .30 & .45 \\
\hline DUDGETUD & & 66 & & & & & &. .46 & & \\
\hline ORIESUP & & .50 & & & .42 & .36 & & & & \\
\hline
\end{tabular}

Notes : Analyse factorielle, rotation varimax, 58 observations ; 10 facteurs expliquant $84 \%$ de la variance totale des 35 variables originales. 
FU2 : Taille de la faculté et orientation vers les études de second et troisième cycle

- nombre de professeurs à temps plein ;

- nombre d'étudiants inscrits au programme de doctorat dans la faculté ;

- pourcentage d'étudiants de l'université qui font des études de second et troisième cycle ;

- au Canada, cette dimension semble caractériser des universités situées dans des régions urbaines (URBAIN) ayant une bonne base industrielle utilisatrices de technologies de pointe (HIGHTECH).

\section{FU3 : Support à l'entrepreneuriat}

- réaction favorable à la création d'entreprises ;

- existence de programmes « COOP » industriels pour les étudiants ;

- existence de cours sur l'entrepreneuriat ;

- universités situées en milieu urbain avec bonne base technologique ;

- universités avec bon financement industriel de la recherche.

\section{FU4 : Orientation industrielle de la faculté}

- importance des bourses et des contrats industriels pour le financement de la recherche ;

- présence d'un comité consultatif dans la faculté ;

- recours pour l'enseignement à plus de vacataires que la moyenne ;

- langue majoritairement francophone (la taille de l'échantillon étant limitée, cette observation linguistique est probablement attribuable au hasard).

\section{FU5 : Recherche et infrastructure}

- niveau moyen des budgets de recherche des professeurs dans la faculté ;

- âge du bureau de transfert de technologie-liaison avec l'industrie (une mesure indirecte de l'importance qu'accorde l'université au soutien à la recherche ;

- inverse du pourcentage des fonds de recherche reçus des conseils de recherche (CRSNG, CRM, CRSH ; cette relation négative est difficile à expliquer) ; 
- importance donnée à l'enseignement lors de l'analyse des demandes de promotion.

Il semble que cette dimension mêle l'enseignement et la recherche, ce qui en complique l'interprétation pratique.

FU6 : BTT proactif

- bureau de transfert de technologie prenant l'initiative de la recherche d'opportunités de transfert au lieu de n'agir qu'en réponse aux demandes des professeurs et chercheurs ;

- inverse de l'importance des publications lors de l'analyse des demandes de promotion des professeurs.

\section{FU7 : Orientation « recherche » de l'université}

- pourcentage du budget total de l'université dépensé pour des activités de recherche ;

- faible charge d'enseignement dans la faculté ;

- université avec des programmes de recherche de taille en médecine et autres domaines en dehors des sciences et du génie (relation suggérée par l'effet limité du financement reçu du CRSNG).

\section{FU8 : BTT important}

- Rôle important du BTT lors de la préparation des contrats de recherche;

- taille du BTT;

- importance relative des fonds reçus des conseils de recherche (CRSNG, CRM, CRSH) ;

- inverse du budget universitaire total par étudiant (relation difficile à expliquer).

\section{FU9 : Ouverture sur l'extérieur}

- laboratoires pouvant être utilisés par les entreprises essaimantes ;

- taille du BTT;

- présence d'un comité consultatif dans la faculté ;

- présence d'un centre d'innovation dans l'université ;

- existence d'un programme COOP dans la faculté ;

- importance donnée aux activités externes dans les décisions de promotion. 


\section{FU10 : Flexibilité}

- possibilité pour un professeur d'avoir un poste permanent à temps partiel ;

- importance de CRSNG dans le financement de la recherche ;

- taille du BTT ;

- inverse de la charge d'enseignement dans la faculté.

Globalement, les dimensions des cultures universitaires déduites de nos variables explicatives sont donc reliées aux activités de recherche des universités (FU1, FU7), au comportement de leur bureau de transfert de technologie (FU2, FU4), à la flexibilité de l'université et à son support à l'entrepreneuriat (FU3, FU6), et à ses contacts avec l'extérieur et avec les milieux financiers (FU5, FU8). Un certain nombre de données manquent dans la banque de données sur les universités, constituant la base de cette analyse, ce qui limite la solidité des résultats. Les dimensions obtenues sont cependant logiques et vont servir de base à l'analyse de la performance des entreprises essaimantes.

\subsection{Les dimensions des " conditions de démarrage " des entreprises essaimantes}

Comme illustré par les statistiques du tableau 2 (page 23), les 36 variables « conditions de démarrage " peuvent être résumées d'une manière assez efficace par 13 « facteurs de démarrage », FD1 à FD13 qui, ensemble, expliquent $74 \%$ de la variance des variables originales. Les autres facteurs potentiels ne contribuant que marginalement (moins que la moyenne) à la variance ont été ignorés. Les dimensions des conditions de démarrage peuvent être inférées à partir des corrélations ou «facteurs de pondération » du tableau 2. Les composantes de chacune de ces dimensions sont notées ci-dessous :

\section{FD1 : Expérience de gestion}

- expérience passée de l'équipe de fondateurs en gestion, finance et marketing.

\section{FD2 : Productivité universitaire}

- fondateur ayant à son crédit plusieurs publications et pouvant bénéficier d'octrois de recherche élevés ;

- non permanent (comme la très grande majorité des entrepreneurs ayant participé à cette étude ont la permanence, la corrélation négative observée avec cette permanence s'explique par les quelques jeunes 
entrepreneurs très actifs en recherche qui n'étaient pas encore permanents au moment de la création de l'entreprise).

\section{FD3 : Planification initiale}

- préparation de plans d'affaire et de plans de marketing au moment de la création de l'entreprise ;

- importance du capital de départ ;

- entrepreneurs ayant une expérience passée de démarrage limitée.

\section{FD4 : Orientation marketing}

- orientation marketing plutôt que technologique de l'entreprise au démarrage (selon le jugement de l'entrepreneur);

- participation financière de l'université (deux cas seulement);

- expérience limitée en $R$ et $D$ de l'équipe de fondateurs (selon leur jugement).

FD5 : Objectif technologique (selon l'entrepreneur)

- objectif initial d'excellence technologique ;

- objectif initial de concentration dans des marchés spécialisés.

FD6 : Objectif initial de croissance

- objectif initial de croissance rapide ;

- entrepreneur productif en recherche avec peu d'expérience en recherche et développement ;

- utilisation dès le début des services d'un administrateur professionnel (deux cas seulement).

\section{FD7 : Expérience industrielle}

- nombre d'années d'expérience industrielle à temps plein.

FD8 : Professeur non titulaire, à l'université depuis moins de temps que la moyenne

FD9 : Expérience gouvernementale

- expérience en passation de contrats avec le gouvernement ;

- importance relative des ventes au secteur public au cours de la première année.

FD10 : Entreprise créée pour être une seconde source dans un marché industriel 
TABLEAU 2

Analyse $F$, conditions de démarrage corrélations $(\leq .30)$ des variables et des dimensions

\begin{tabular}{|c|c|c|c|c|c|c|c|c|c|c|c|c|c|}
\hline $\begin{array}{l}\text { Factour } \\
\text { Vartable }\end{array}$ & FD1 & FD2 & FD3 & FD4 & FD5 & FD6 & FD7 & FD8 & FO9 & FO10 & FD11 & FO12 & FD13 \\
\hline EMPIND & & & & & & & .78 & & & & & & \\
\hline POSITION & & & & & & & & .78 & & & & & \\
\hline PERMAN & & .89 & & & & & & & & & & & \\
\hline ANNEIS & & & & & & & &. .77 & & & & & \\
\hline QUITUNI? & .30 & & & & .66 & & & & & & & & \\
\hline TECHUEE & & & 46 & & & & & & .52 & & & & \\
\hline BRENUN &. .30 & & & & & & & & & & 30 & 38 & \\
\hline FONDAT & & & & & & & & & & & .77 & & \\
\hline COVIO & & & & & & & & & .73 & & & & \\
\hline STMED & & & & & & .30 & & & &. .47 & .31 & & .31 \\
\hline OBNECTIF 1 & & & & & & .60 & & & .30 & & & & \\
\hline OBNECTIF 2 & & & & & .84 & & & & & & & & \\
\hline OBNECTIF 3 & & & & & .43 & &. .45 & .32 & & & & & \\
\hline OBNECTIF 4 & & & & & & & & & & .84 & & & \\
\hline OBUECTIF 5 & & & & & .77 & & & & & & & & \\
\hline PLANAFF & & & .70 & & & & & & & & & & \\
\hline PLANMAK & & & .52 & & & & & & &. .31 & & .31 & \\
\hline ORENTD & & & & .53 & & .30 & & & & .36 & & & \\
\hline ENCACEM & & & & & & & & & & & & .86 & \\
\hline XGESTIONA & .81 & & & & & & & & & & & & \\
\hline XFNNANCE & .84 & & & & & & & & & & & & \\
\hline XMARKET & .76 & & & & & & & & & & & & \\
\hline$X \in D$ & & & &. .36 & & .59 & & & & & & & \\
\hline XVIECON & .37 & & & & & & & & .64 & & & & \\
\hline XDEMAR & & & -.69 & & & & & & & & & & \\
\hline CAPOEP & & & .55 & & & & & & & & .31 &. .30 & .30 \\
\hline ANEXPIND & & & & & & & .75 & & & & & & \\
\hline PUBL1 & & .53 & & & & .56 & & & & .30 & & & \\
\hline FNRECHI & & .93 & & & & & & & & & & & \\
\hline UNIVCAP & & & & .79 & & & & & & & & & \\
\hline FONDCAP & & & & & & & & & & & & .34 &. .62 \\
\hline FONDCTRD & & & &. .72 & & & & & & & & & \\
\hline GESTPROF & & & & & & .72 & & & & & & .38 & \\
\hline TEMPSO & & & 38 & & & & .42 & & & & 48 & & \\
\hline RETARD & & & & & & & & & & & & & .81 \\
\hline AUTFOND & & & & .48 & & & & & & & .51 & & \\
\hline
\end{tabular}

Notes : Analyse factorielle, rotation varimax, 58 observations; 13 facteurs expliquant $74 \%$ de la variance totale des 36 variables originales. 


\section{FD11 : Grande équipe avec des fondateurs externes}

- nombre de fondateurs ;

- pourcentage de fondateurs non universitaires.

\section{FD12 : Engagement personnel}

- engagement personnel de l'entrepreneur au moment de la création de l'entreprise ;

- financement personnel investi dans l'entreprise ;

- utilisation limitée de gestionnaires professionnels au moment du démarrage.

\section{FD13 : Démarrage financé par l'extérieur}

- capital de départ plus élevé que la moyenne ;

- investissement personnel de l'entrepreneur inférieur à la moyenne ;

- délais de mise en vente (ce qui indique que l'entreprise a commencé ses opérations par les activités de recherche et développement requises pour mettre en marché son premier produit).

Les dimensions qui caractérisent les conditions de démarrage des entreprises dans l'échantillon représentent donc la composition et l'expérience de l'équipe de fondateurs (FD1, FD3, FD6, FD9, FD11), les objectifs et stratégie de démarrage (FD4, FD5, FD7, FD10, FD13), le profil universitaire du fondateur principal (FD2, FD8) et l'engagement personnel de ce fondateur (FD5, FD12, FD13).

Ces dimensions ne sont pas complètement indépendantes de la culture universitaire : certaines universités attirent certains types de personnes et l'environnement universitaire affecte le comportement des professeurs. Les corrélations significatives observées entre les dimensions de démarrage et les dimensions universitaires sont les suivantes (tableau 3, page 26) :

\section{FD1 : Expérience en gestion}

- corrélée négativement avec FU7, orientation « recherche » de l'université.

Ceci est en accord avec l'observation usuelle du peu de connaissances en gestion des scientifiques.

FD2 : Productivité universitaire

- corrélée positivement avec l'orientation « recherche » de l'université et de la faculté. 


\section{FD5 : Objectif technologique}

- corrélée négativement avec la flexibilité montrée par l'université ;

- corrélée négativement avec l'importance du BTT comme gestionnaire des contrats de recherche dans l'université.

Cette relation est difficile à expliquer, mais pourrait être le fait des BTT encourageant la recherche à l'université plutôt que dans une entreprise essaimante à l'extérieur.

\section{FD6 : Objectif de croissance}

- corrélée positivement (faiblement) avec l'importance du BTT de l'université.

\section{FD7 : Expérience industrielle de l'entrepreneur}

- corrélée (faiblement) avec le support à l'entrepreneuriat offert par les universités, ces universités étant attirantes pour les jeunes chercheurs de l'industrie.

FD8 : Professeur non titulaire, à l'université depuis peu de temps

- corrélée positivement avec l'orientation industrielle de la faculté et son ouverture vers l'extérieur.

Ceci concorde avec le désir de certains professeurs en génie de développer rapidement leurs liens avec le monde industriel.

FD9 : Expérience gouvernementale de l'entrepreneur

- corrélée positivement avec l'orientation « recherche » et industrielle de la faculté et de l'université ;

- corrélée positivement avec la taille de la faculté et son orientation vers les études supérieures ;

- corrélée négativement (relation faible) avec le support à l'entrepreneuriat offert par l'université, ce qui est surprenant et pourrait être avoir pour cause la petite taille de l'échantillon.

FD10 : Entreprise créée comme « seconde source »

- corrélée négativement avec l'orientation « recherche » de l'université.

Cette relation négative n'est pas surprenante, une stratégie de « seconde source » étant orientée vers la demande plutôt que vers le leadership technologique.

FD11 : Grande équipe de fondateurs, avec fondateurs externes

- corrélée positivement avec l'orientation vers les études supérieures de la faculté ; 
- corrélée positivement avec le support à l'entrepreneuriat dans l'université.

\section{FD12 : Engagement personnel}

- corrélée positivement avec la présence d'un BTT proactif.

Les conseils du BTT encouragent probablement ceux qui désirent vraiment créer une entreprise à le faire sérieusement.

\section{FD13 : Financement de départ extérieur}

- corrélée positivement avec l'importance du BTT dans l'université, le BTT étant souvent un «facilitateur » dans les contacts avec le monde des affaires à l'extérieur de l'université.

La petite taille de l'échantillon sur lequel est basé cette analyse limite le nombre et le niveau de signification des relations observées. La qualité des inférences sur les relations entre dimensions « culture universitaire » et dimensions « conditions de départ » est donc limitée. La concordance de certains résultats avec ce qui est décrit dans la littérature augmente cependant la validité de l'ensemble des relations observées.

\section{TABLEAU 3}

\section{Corrélation des facteurs universitaires et de démarrage}

\begin{tabular}{|l|l|l|l|l|l|l|l|l|l|l|}
\hline & FU1 & FU2 & FU3 & FU4 & FU5 & FU6 & FU7 & FU8 & FU9 & FU10 \\
\hline FD1 & & & & & & & .24 & & & \\
\hline FD2 & & .21 & & & .28 & & & & & \\
\hline FD3 & & & & & & & & & & \\
\hline FD4 & & & & & & & & & & \\
\hline FD5 & & & & & & & & .24 & &. $.25^{\circ}$ \\
\hline FD6 & & & & & & & & .19 & & \\
\hline FD7 & & & .19 & & & & & & & \\
\hline FD8 & & & & .25 & & & & & & \\
\hline FD9 & .20 & .27 & .22 & $.33^{\circ}$ & & & & & & \\
\hline FD10 & .20 & & & .19 & & & & & & \\
\hline FD11 &. .19 & .28 & .35 &. .19 & & & & & & \\
\hline FD12 & & & & & & & & .22 & &. $.31^{\circ}$ \\
\hline FD13 & & & & & & & & $.29^{\circ}$ & & \\
\hline
\end{tabular}

Notes : Analyse factorielle, rotation varimax, 58 observations ; 10 facteurs expliquant $84 \%$ de la variance totale des 35 variables originales. 


\subsection{Relation entre les dimensions " culture universitalre " et "conditions de départ ", la performance des entreprises et l'évolution de la carrière de l'entrepreneur universitaire}

L'une des propositions motivant cette recherche concerne l'effet à court terme sur l'entreprise et sur l'entrepreneur de l'environnement universitaire et des conditions de démarrage de l'entreprise.

L'évolution de l'entreprise est évaluée selon le « succès » et la structure financière. Comme dans bon nombre d'études sur les entreprises émergentes, le niveau annuel des ventes est l'un des critères de succès utilisé (ici, pour une analyse à court terme, ventes annuelles au cours des cinq premières années, CS1 à CS5, en dollars constants). L'évaluation subjective par l'entrepreneur du comportement présent de son entreprise (COBIEN) est aussi un autre critère. D'autres mesures de succès ont été suggérées dans la littérature, certaines quantitatives (survie à long terme, valeur boursière, part de marché, profitabilité, taux de croissance des ventes, nombre d'employés), d'autres descriptives (satisfaction des objectifs, visibilité, acceptation sociale) (Simyar et Doutriaux, 1987). Dans le cas de petites entreprises émergentes, ces mesures sont soit inexistantes (valeurs boursière), très subjectives (satisfaction des objectifs, acceptation sociale), difficiles à estimer d'une manière consistante (profitabilité), ou très erratique (taux de croissance). Le niveau des ventes a l'avantage de ne pas être confidentiel, d'être généralement disponible et facile à mesurer.

La structure financière de l'entreprise est évaluée par le niveau de contrôle conservé par les fondateurs (FONDCTRM), par le niveau de contrôle détenu par les investisseurs de capital de risques (CAPRISQ) et par le ratio actuel de la dette sur capital (RATIODCAP).

L'évolution de la carrière de l'entrepreneur universitaire principal est mesurée par sa position actuelle par rapport à l'université (AVECUNI), par le temps passé jusqu'à maintenant sur les affaires de l'entreprise (TEMPSM) et par sa productivité universitaire au cours des $2^{\mathrm{e}}$ et $3^{\mathrm{e}}$ années qui ont suivi la création de l'entreprise (PUBLI2, FINRECH2).

Chacune de ces variables est décrite en détail dans l'annexe.

Comme précédemment, l'évaluation des relations entre la performance des entreprises et les dimensions « culture universitaire » et « conditions de démarrage » est basée sur l'observation de corrélations de Pearson (tableau 4). Dans ce tableau, pour montrer la continuité de certains effets sur le niveau des ventes, on a reproduit certaines corrélations jusqu'à un seuil de signification plus élevé que de coutume (jusque $25 \%$ ) lorsque les corrélations avec les ventes d'une année voisine étaient significatives aux seuils habituels. 


\subsubsection{Niveau annuel des ventes au cours des années 1 à 5}

Selon l'analyse de corrélation du tableau 4 (page suivante), plusieurs facteurs caractérisant l'environnement universitaire sont reliés significativement au niveau des ventes au cours de certaines des cinq premières années des entreprises :

- taille de la faculté et orientation vers les études supérieures (FU2);

- recherche et infrastructure (FU5);

- ouverture vers l'extérieur (FU9).

Comme cela a été noté précédemment, certaines des caractéristiques représentées par ces facteurs incluent l'âge et la taille du BTT, le nombre d'étudiants de doctorat et de professeurs dans la faculté, le montant des budgets de recherche alloué par professeur.

L'effet positif sur le niveau des ventes des variables caractérisant les conditions de départ est aussi mis en évidence par les données du tableau 4 :

- effort de planification fait au moment de la création de l'entreprise (FD3) ;

- objectif technologique / orientation technologique de l'entreprise au moment du démarrage (FD5) ;

- expérience industrielle passée de l'entrepreneur principal (FD7, première année seulement) ;

- grande équipe de démarrage avec des fondateurs externes (FD11).

Il est important de noter la relation négative existant entre la variable FD12 (engagement personnel) et le niveau des ventes : dans la mesure où il limite le recours à des gestionnaires professionnels et l'apport de capitaux externes, cet engagement personnel peut avoir un effet négatif sur la croissance des ventes.

\subsubsection{Contrôle de l'entreprise et caractéristiques financières}

Pour croître, la majorité des entreprises doivent avoir recours à des capitaux externes; leurs fondateurs doivent donc généralement accepter de partager la propriété de l'entreprise et de réduire leur niveau de contrôle. Dans l'échantillon, le niveau de contrôle encore aux mains des fondateurs en 1990 (FONDCTRM) est corrélé positivement avec l'orientation industrielle de la faculté (FU4), et négativement avec son ouverture sur l'extérieur : la présence d'un comité consultatif et l'ouverture de la faculté sur l'extérieur favorise les contacts avec des investisseurs éventuels, alors que les contrats de recherche industriels passés par la faculté semblent avoir un effet contraire, ces contacts industriels réduisant 
TABLEAU 4

Corrélations des indicateurs de performance avec les facteurs universitaires et de démarrage

\begin{tabular}{|c|c|c|c|c|c|c|c|c|c|c|c|c|c|}
\hline Performance & CS1 & cS2 & $\operatorname{cs3}$ & cs4 & Cs5 & $\begin{array}{l}\text { FOND } \\
\text { CTRM }\end{array}$ & $\begin{array}{l}\text { CAP } \\
\text { RISO }\end{array}$ & $\begin{array}{l}\text { PUBLI } \\
2\end{array}$ & $\begin{array}{l}\text { FINAE } \\
\text { CH2 }\end{array}$ & $\begin{array}{l}\text { RATIO } \\
\text { DCAP }\end{array}$ & $\begin{array}{l}\text { AVEC } \\
\text { UNI }\end{array}$ & $\begin{array}{l}\text { TEM } \\
\text { PSM }\end{array}$ & $\begin{array}{c}\text { COBI } \\
\text { EN }\end{array}$ \\
\hline \multicolumn{14}{|l|}{$\begin{array}{l}\text { Factours } \\
\text { univereitairee }\end{array}$} \\
\hline Fu1 & & & & & & & & -23 & $-.19 \%$ & & & & \\
\hline Fu2 & $.27+$ & 23 & $17 *$ & 17* & & $-18 *$ & & & .26 & 19 & & .20 & \\
\hline Fus & & & & & & & $33^{\circ}$ & & & & & $.30+$ & \\
\hline Fus & & & & & & .24 & & & & $.32+$ & & & \\
\hline Fus & 19* & 23 & $19 *$ & $.22 *$ & & & & & & & & & \\
\hline \multicolumn{14}{|l|}{ Fus } \\
\hline \multicolumn{14}{|l|}{ Fut } \\
\hline Fus & & & & & & & & 22 & 26 & & & & \\
\hline$F U_{0}$ & $27^{\circ}$ & .23 & $17 *$ & & & .20 & & & & & $-.27+$ & $.29+$ & 21 \\
\hline \multirow{2}{*}{\multicolumn{14}{|c|}{$\begin{array}{l}\text { FU10 } \\
\text { Faotoure do } \\
\text { démarrage }\end{array}$}} \\
\hline & & & & & & & & & & & & & \\
\hline \multicolumn{14}{|l|}{ FD1 } \\
\hline FDR & & & & & & & & $.38^{\circ}$ & $.31^{\circ}$ & & & & $-.26+$ \\
\hline FDO & & 21* & $.36+$ & $42^{\circ}$ & $19 *$ & & & & & & & $43^{\circ}$ & 21 \\
\hline FOA & & & & & &. $.33^{\circ}$ & & -.26 & .24 & & $-.18 *$ & 10 & \\
\hline FDS & & $39^{\circ}$ & $43^{\circ}$ & $40^{\circ}$ & & & $18 *$ & & 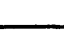 & & $. .30+$ & $.29+$ & \\
\hline FDO & & & & & & & & $.52^{\circ}$ & $.31+$ & & .21 & & $\cdots$ \\
\hline FOP & $.33+$ & & & & & & & & &. .25 & & & \\
\hline FOS & 81 & & & & & & & & & & $. .26+$ & & \\
\hline$F D O$ & & & & & & & $-.27+$ & & & & & & .19 \\
\hline FD10 & & & & & & 20 & & & & & & & \\
\hline FD11 & .22 & & & $.33+$ & & & $30+$ & & .21 & & & $37^{\circ}$ & \\
\hline FD12 & & $-.33^{\circ}$ & $-.33^{\circ}$ & & & &. .20 & & & & 23 & & \\
\hline $\mathrm{FD}_{13}$ & & & & & &. $.41^{\circ}$ & $32^{\circ}$ & $40^{\circ}$ & $60^{\circ}$ & & & & \\
\hline
\end{tabular}

Notes : Corrélations de Pearson, 33 à 58 observations ; seuil de signification : ${ }^{*} 1 \%+5 \%$; autrement $15 \%$ (et, exceptionnellement, \# indique entre $15 \%$ et $25 \%$ )

probablement la liberté du chercheur-entrepreneur. La corrélation négative observée entre le niveau de contrôle conservé par l'entrepreneur et FD4 (orientation marketing) confirme le besoin de financement externe exigé par une stratégie « marketing ».

Le niveau de contrôle maintenant aux mains d'investisseurs externes (CAPRISQ) est relié positivement au support à l'entrepreneuriat offert par l'université (cette dimension inclut la variable «INVRISQ » mesurant les contacts financiers externes du bureau de transfert de technologie), à l'orientation technologique des entreprises, à la présence de fondateurs externes et au financement externe reçu au moment du démarrage. Ce niveau de contrôle semble être affecté négativement par l'engagement personnel de l'entrepreneur dans son entreprise et par son expérience gouvernementale. Ces observations sont congruentes avec 
les analyses d'entreprises émergentes rapportées dans la littérature : effet négatif sur le financement (et sur la croissance) d'un entrepreneur désirant tout contrôler, effet positif d'une bonne base technologique et d'une ouverture sur l'extérieur.

Le niveau d'endettement des entreprises en 1990, ou au bout de cinq ans si elles ont été créées avant 1985, (RATIODCAP) ne varie pas de la même manière que l'investissement externe au cours de la même période, les taux d'autofinancement variant entre les entreprises en fonction de leur succès (RATIODCAP et FONDCTRM ne sont pas corrélés avec le niveau des ventes ; CAPRISQ a une faible corrélation positive avec ces ventes). Ce niveau d'endettement est positivement corrélé avec l'orientation industrielle de l'université et négativement avec l'expérience industrielle passée de l'entrepreneur, une contradiction qui n'est qu'apparente. En effet, l'orientation industrielle de la faculté facilite probablement l'accès au financement externe, alors que l'expérience industrielle passée de l'entrepreneur peut l'encourager à réduire les risques en évitant un endettement trop important.

\subsubsection{Statut et productivité universitaire de l'entrepreneur}

Les entrepreneurs qui, quelques années après la création de l'entreprise, étaient encore employés par leur université (AVECUNI, $74 \%$ des entrepreneurs dans l'échantillon) sont généralement des professeurs titulaires à l'université depuis longtemps et qui avaient peu d'objectifs de marketing ou d'innovation technologique (FD4, FD5) au moment du démarrage. Les commentaires reçus au cours d'entrevues avec 26 entrepreneurs permettent d'expliquer ces résultats : $40 \%$ étaient des professeurs titulaires désirant terminer leur carrière à l'université, mais qui cherchaient un nouveau défi à relever, une nouvelle activité à mener parallèlement à leur vie universitaire ; au moment de la création de leur entreprise, ils n'ont pas toujours d'objectifs précis, ni l'intention de quitter l'université.

Logiquement, les entrepreneurs encore à l'université (AVECUNI) passent moins de temps sur les affaires de leur entreprise (TEMPSM) que les autres (33\% de leur temps en moyenne comparé avec $73 \%$ pour ceux qui ont quitté l'université, corrélation négative significative entre AVECUNI et TEMPSM). Ce sont les entrepreneurs ayant fait une bonne planification initiale (FD3) avec une équipe de fondateurs en majorité externes (FD11), ayant des objectifs technologiques et issus d'université ouvertes sur l'extérieur, qui passent actuellement le plus de temps sur les affaires de leur entreprise. Leurs entreprises sont aussi les plus grosses, [TEMPSM étant fortement corrélé avec le niveau des ventes (CS1,..., CS5) et celles qui ont reçu le plus de financement externe (corrélation 
significative positive avec CAPRISQ et négative avec FONDCTRM). Il faut noter que les entrepreneurs ayant quitté l'université ont des ventes significativement plus élevées que les autres pendant les trois premières années d'opération, mais que la différence semble disparaître après, soit parce que beaucoup d'entreprises ont maintenant un gestionnaire professionnel (en charge de $14 \%$ de la gestion en moyenne, comparé à $4 \%$ au moment de la création), soit parce que l'échantillon ne contient pas suffisamment d'entrepreneurs ayant quitté leur université ( $26 \%$ de l'échantillon), ou encore parce que l'échantillon est biaisé en faveur de ceux qui ont réussi.

La productivité universitaire des entrepreneurs au cours des $2^{e}$ et $3^{e}$ années qui ont suivi la création de leur entreprise semble être beaucoup plus affectée par leurs caractéristiques personnelles et les décisions prises au moment du démarrage que par l'environnement universitaire. En effet, le nombre de publications (PUBLI2) et le niveau des fonds de recherches obtenus (FINRECH2) sont corrélés très positivement avec la productivité universitaire de départ (FD2 et FD6, un objectif de croissance lui-même corrélé avec le nombre de publications de l'entrepreneur avant le démarrage), le financement externe (FD13), ainsi qu'avec l'importance du BTT de l'université (FU8, une mesure indirecte de l'importance donnée à la recherche appliquée par l'université). Le recours à des sources de financement externe caractérisent donc les chercheurs les plus productifs. La productivité universitaire post démarrage des entrepreneurs est aussi corrélée négativement avec l'orientation marketing initiale choisie pour l'entreprise (FD4, donc les entreprises ayant des liens avec la recherche moins forts que dans le cas d'une orientation technologique), et avec l'orientation « recherche » de l'université (FU1) ; cette dernière relation est difficile à expliquer et pourrait résulter d'une dichotomie entre la recherche théorique pure et la recherche appliquée. Il faut cependant noter que s'il n'y a pas de corrélation entre le niveau des ventes et les niveaux de publication ou de bourses de recherche avant la création des entreprises, les niveaux de publication et des bourses après la création sont négativement corrélés (significativement) avec les ventes des entreprises au cours de leurs deux premières années; cette relation temporaire s'explique soit par le démarrage difficile des entreprises créées par des chercheurs actifs, soit par des revenus réduits pendant le développement final des produits préparés par ces chercheurs spécialement actifs. Une étude à plus long terme serait nécessaire pour analyser les différences de succès des entreprises créées par des chercheurs actifs et des moins actifs.

Les entrepreneurs satisfaits du succès de leur entreprise (COBIEN) ont fait plus de planification initiale que les autres (FD3), viennent d'université ouvertes sur l'extérieur (FU9), ont un peu plus de contrats gouvernementaux que les autres (FD9), mais sont probablement un peu moins productifs du point de vue universitaire (FD2). Leur taux de satisfaction n'est que faiblement corrélé 
(positivement) avec le niveau des ventes; il est cependant corrélé négativement (significativement) avec le fait d'être encore à l'université (AVECUNI), montrant clairement une fois de plus que la position de « professeur-entrepreneur » n'est pas des plus faciles.

\section{Discussion des résultats et conclusions}

Cette analyse empirique apporte un certain nombre de réponses aux questions posées après l'exposé du cadre conceptuel sur lequel est basée cette étude.

Dans l'échantillon, les conditions de démarrage des entreprises varient en effet selon les universités et dépendent donc de la culture universitaire locale :

- Les entrepreneurs issus d'universités orientées vers la recherche sont plus actifs que la moyenne en recherche et ont généralement plus d'expérience en contrats gouvernementaux que ceux qui viennent d'universités où se fait peu de recherche, mais ils ont moins d'expérience en gestion que ces derniers. Et les entrepreneurs issus d'universités de recherche avec une orientation industrielle ont tendance à être plus jeunes que les autres.

- Les universités supportant activement l'entrepreneuriat (favorables à l'essaimage, avec programmes COOP, offrant des cours d'entrepreneuriat, etc.) tendent à avoir des entreprises avec plus de fondateurs incluant des personnes non liées à l'université et plus de fondateurs ayant une expérience professionnelle passée à temps plein dans l'industrie.

- Les universités avec un BTT important et actif ont plus d'entreprises dirigées par des gestionnaires professionnels que les autres, et font appel à des sources de financement externes.

Selon l'échantillon, les entreprises qui croissent le plus rapidement (cinq premières années):

- Viennent de grosses facultés ouvertes vers l'extérieur ; elles ont un pourcentage d'étudiants de second et troisième cycles et un budget moyen de recherche par professeur élevé et sont situées dans des universités avec un BTT bien établi.

- Ont été créées par des équipes de fondateurs avec des personnes de l'extérieur qui ont fait un bon effort de planification au moment du démarrage et elle ont une orientation et des objectifs d'excellence technologique. 
Il est intéressant de noter que l'ouverture de la faculté sur l'extérieur ne semble pas affecter directement les conditions de démarrage des entreprises, mais elle a un impact sur le financement des entreprises émergentes, donc sur leur niveau d'activité et sur leurs ventes. Ce financement externe, ou plutôt le pourcentage de contrôle aux mains d'investisseurs externes, dépend aussi de l'orientation technologique des entreprises et du support à l'entrepreneuriat dans l'université même.

Cette étude ne permet pas de dire qui, des chercheurs actifs et des moins actifs, créent le plus d'entreprises. Mais, parmi les entrepreneurs, la productivité universitaire avant la création de l'entreprise ne semble pas être corrélée avec le niveau des ventes au cours des premières années. La productivité universitaire après la création de l'entreprise est fortement corrélée avec la productivité avant la création et avec l'utilisation de fonds externes. Même si, à court terme (trois ans), on observe une corrélation négative entre la productivité universitaire après la création et les ventes, le financement supplémentaire obtenu par les chercheurs actifs devrait, à moyen terme, donner des entreprises à croissance rapide. Globalement, il semble donc que, pour ceux qui décident de devenir entrepreneurs, productivité universitaire et succès dans les affaires vont de pair.

De plus, il semble que le BTT fasse généralement une différence: les entreprises venant d'universités ayant un BTT important et proactif ont tendance à être plus grosses que les autres, à avoir une orientation technologique plus marquée, à utiliser plus de financement externe, à avoir une plus grosse équipe de fondateurs et, globalement, à avoir un plus gros potentiel de croissance que les entreprises venant d'autres universités. En réalité, ces effets sont-ils dus aux actions du BTT ou à d'autres caractéristiques universitaires (ouverture vers l'extérieur et contrats industriels), le BTT étant aussi la résultante de ces autres caractéristiques ? Plus de recherches sont nécessaires pour répondre à cette question.

Cette étude a confirmé l'importance de certaines caractéristiques des universités, des conditions de démarrage et des caractéristiques des entrepreneurs sur le développement des entreprises issues des universités. La qualité de l'analyse est malheureusement limitée par la difficulté d'obtenir un échantillon vraiment représentatif de l'essaimage universitaire au Canada : la population n'étant pas bien connue, il est difficile de mesurer la représentativité de l'échantillon utilisé et donc d'évaluer la validité externe des conclusions. Les résultats sont cependant consistants avec ce que l'on trouve dans la littérature. Ils correspondent donc fort probablement à la réalité et contribuent à la connaissance du processus d'essaimage universitaire. 


\section{Bibliographie}

Association DES UNIVERSITES ET COLLĖges DU CANADA ( 1989), Universities Telephone Directory, Ottawa.

BAGBY, J.W. et K. RAO (1990), « Strategic advantages in management of university related start-ups : issues and challenges », The Pennsylvania State University Center for Enterprise Creation and Growth, W.P. Series on Entrepreneurship, 90-1E.

BIRCH, D. (1979), The Job Generation Process, MIT, Cambridge, MA.

BROWN, W.S. (1984), «A proposed mechanism for commercializing university technology », Frontiers of Entrepreneurship Research, J.A. Hornaday et alii éds, Babson College, p. 136-143.

DILL, D.D. (1990), «University/industry research collaboration : an analysis of interorganizational relationships », R\&D Management, vol. 20, $\mathrm{n}^{\circ} 2$, p. 123-129.

DoutRIAUX, J. (1987), « Growth pattern of academic entrepreneurial firms », Journal of Business Venturing, vol. 2, $n^{\circ} 4$, p. 285-297.

DoutriauX, J. (1992), «Emerging high-tech firms : how durable are their comparative start-up advantages ? », à paraître, Journal of Business Venturing.

DoutriauX, J. et B. Peterman (1982), « Technology transfer and academic entrepreneurship at canadian universities », rapport de recherche, Technological Innovation Studies Program, Technology Branch, Department of Trade, Industry and Commerce, Ottawa. Pour un court résumé, voir J. Doutriaux. and B. Peterman, «Technology transfer and academic entrepreneurship », K. Vesper (éd.), Frontiers of Entrepreneurship Research, Babson College, Wellesley, p. 430-448.

ENRos, P. et M. Farley (1986), « University offices for technology transfer : toward the service university », Science Council of Canada, Ottawa, Document de travail, avril.

Louis, K.S., D. Blumenthal, M.E. Gluck et M.A. Stoto (1989), « Entrepreneurs in academy : an exploration of behavior among life scientists $"$, Administrative Science Quarterly, vol. 34, p. 110-131.

MCMULlaN, W.E. et K MELNYK (1988), « University innovation centres and academic venture formation », $R \& D$ Management, vol. $18, \mathrm{n}^{\circ} 1, \mathrm{p} .5-12$.

MunsChe, P. (1990), «Waiting for gatorade », University of Toronto Bulletin, le 8 janvier, p. 20.

ROGERS, E. (1986), "The role of the research university in the spin-off of hightechnology companies », Technovation, vol. 4, p. 5-12. 
Olofsson, C., G. Reiberger, P. Tovman et C. WAHLbin (1987), « Technology-based new ventures from Swedish universities : a survey », Frontiers of Entrepreneurship Research, Babson College.

Olofsson, C. et C. WAHLBIN (1984), « Technology-based new ventures from technical universities : a Swedish case ", Frontiers of Entrepreneurship Research, K.Vesper et alii, (éds), Babson College.

SEGAL, N.S. (1986), « Universities and technological entrepreneurship in Britain : some implications of the Cambridge phenomenon », Technovation, vol. 4, p. 189-204.

SIMYA, F. et J. DOUTRIAUX (1987), « Use of accounting ratios on measuring success : the high-tech entrepreneurial firms in Canada », Actes, Conference of the Canadian Academic Accounting Association, McMaster University, et document de travail 87-40, Faculté d'administration, Université d'Ottawa.

VAN DiERDoncK, F. et K. DeBACKERE (1988), « Academic entrepreneurship at Belgian universities », R\&D Management, vol. 18, p. 341-353.

CONSEIL DES SCIENCES DU CANADA (1988), Winning in a World Economy : UniversityIndustry Interaction and Economic Renewal in Canada, Ottawa, Canada, avril.

STATIstics CANADA (1989), Science and Technology Indicators, nº 88-002, mars. 


\section{ANNEXE}

Liste complète et codification des variables utilisées dans cette étude

\section{Codification :}

b binaire, oui $=1$, non $=0$;

o ordinal, pas important, peu $=1$, très important, beaucoup $=3$;

n nominal, requis $=1$, optionnel $=2$, non $=3$;

$\%$ pourcentage ;

v valeur, niveau ;

\section{Culture universitaire et environnement}

\begin{tabular}{|c|c|c|}
\hline Nom & $\begin{array}{l}\text { Codifi- } \\
\text { cation }\end{array}$ & Description \\
\hline INST & b & faculté affiliée à un institut de recherche universitaire ; \\
\hline RESEAU & b & liens formels avec des instituts de recherche externes ; \\
\hline COMCONS & b & faculté avec un comité consultatif ; \\
\hline PUBL & $\%$ & $\begin{array}{l}\text { importance relative des publications lors } \\
\text { des décisions de promotion; }\end{array}$ \\
\hline ACTEXT & $\%$ & $\begin{array}{l}\text { importance relative des activités externes } \\
\text { lors des décisions de promotion; }\end{array}$ \\
\hline EVALENS & $\%$ & $\begin{array}{l}\text { importance relative des évaluations de l'enseignement } \\
\text { lors des décisions de promotion; }\end{array}$ \\
\hline CRSENTR & $\mathrm{n}$ & cours en entrepreneurship offerts aux étudiants ; \\
\hline COOP & b & programme coopératif dans la faculté ; \\
\hline BTT & b & $\begin{array}{l}\text { l'université a un bureau de transfert } \\
\text { de technologie/liaison industrielle ; }\end{array}$ \\
\hline INNOV & b & l'université a un centre d'innovation ; \\
\hline ACCESLAB & b & $\begin{array}{l}\text { les entreprises essaimantes peuvent avoir accès } \\
\text { aux laboratoires ; }\end{array}$ \\
\hline BUDRPROF & $\mathbf{v}$ & budget de recherche moyen par professeur,1989; \\
\hline CHARGENS & $\mathbf{v}$ & $\begin{array}{l}\text { charge moyenne d'enseignement des professeurs } \\
\text { à temps plein (nombre de cours/an); }\end{array}$ \\
\hline ENSTPL & $\%$ & $\begin{array}{l}\text { pourcentage des cours de la faculté donnés } \\
\text { par le corps enseignant à temps plein ; }\end{array}$ \\
\hline PHDSPR & $\mathbf{v}$ & nombre moyen d'étudiants de doctorat par professeur ; \\
\hline PHDS & $\mathbf{v}$ & nombre d'étudiants de doctorat dans la faculté ; \\
\hline NTPFAC89 & $\mathbf{v}$ & $\begin{array}{l}\text { nombre total de professeurs à temps plein } \\
\text { dans la faculté ; }\end{array}$ \\
\hline
\end{tabular}




\begin{tabular}{|c|c|c|}
\hline OCTROISR & $\%$ & $\begin{array}{l}\text { pourcentage de la recherche financée } \\
\text { par les grandes agences académique ; }\end{array}$ \\
\hline INDUST & $\%$ & $\begin{array}{l}\text { pourcentage de la recherche financée } \\
\text { par des bourses et contrats industriels ; }\end{array}$ \\
\hline TPARTIEL & b & $\begin{array}{l}\text { entrepreneurs et consultants peuvent avoir } \\
\text { un poste permanent à temps partiel ; }\end{array}$ \\
\hline SUPPORTF & $\mathrm{b}$ & $\begin{array}{l}\text { l'université regarde favorablement } \\
\text { les entreprises essaimantes ; }\end{array}$ \\
\hline LANGUE & & $\begin{array}{l}\text { langue majoritaire de l'université, } \\
\text { français = } 1 \text {, anglais = } 0 \text {; }\end{array}$ \\
\hline URBAIN & b & $\begin{array}{l}\text { université située dans une grande agglomération } \\
\text { (au moins } 500000 \text { habitants); }\end{array}$ \\
\hline HIGHTECH & b & $\begin{array}{l}\text { région très industrialisée et/ou considérée } \\
\text { « high-tech »; }\end{array}$ \\
\hline EMPBTT & $\mathbf{v}$ & nombre d'employés (temps plein et équivalent), BTT ; \\
\hline CONBTT & $\%$ & $\begin{array}{l}\text { contrats de recherche universitaire négociés } \\
\text { par le BTT (par rapport au nombre total); }\end{array}$ \\
\hline BTTINIT & $\%$ & $\begin{array}{l}\text { importance relative des initiatives des employés } \\
\text { du BTT pour l'identification d'innovations } \\
\text { avec bon potentiel (visites aux professeurs, } \\
\text { aux laboratoires, séminaires); }\end{array}$ \\
\hline INVRISQ & b & $\begin{array}{l}\text { excellente coopération avec des entreprises } \\
\text { de capitaux de risques; }\end{array}$ \\
\hline AGEBTT & $\mathbf{v}$ & âge du bureau de transfert de technologie, en années ; \\
\hline PROACT & $\%$ & $\begin{array}{l}\text { pourcentage des contrats de recherche négociés } \\
\text { par le BTT et faisant suite aux visites de ses employés } \\
\text { aux professeurs et laboratoires ; }\end{array}$ \\
\hline REACT & $\%$ & $\begin{array}{l}\text { pourcentage des contrats de recherche négociés } \\
\text { par le BTT et faisant suite aux demandes } \\
\text { de professeurs ou de clients externes; }\end{array}$ \\
\hline BUDRUNI & $\%$ & budget de recherche sur le budget total de l'université ; \\
\hline CRSNGSH & $\%$ & $\begin{array}{l}\text { part du budget total de recherche de l'université } \\
\text { provenant du Conseil de recherche en sciences } \\
\text { naturelles et en génie du Canada; }\end{array}$ \\
\hline BUDGETUD & $\mathbf{v}$ & budget total de l'université par étudiant ; \\
\hline ORIESUP & $\%$ & $\begin{array}{l}\text { orientation « études supérieures » de l'université, } \\
\text { rapport du nombre d'étudiants de } 2^{\circ} \text { et } 3^{\circ} \text { cycle } \\
\text { sur le nombre total d'étudiants. }\end{array}$ \\
\hline
\end{tabular}

\section{Conditions de démarrage}

$\begin{array}{lll}\text { EMPIND } & \text { b } & \text { expérience industrielle passée à temps plein ; } \\ \text { POSITION } & \text { niveau de scolarité de l'entrepreneur universitaire } \\ \text { au moment du démarrage }: \text { professeur titulaire }=1, \\ \text { professeur agrégé }=2, \text { autre }=3 ;\end{array}$




\begin{tabular}{|c|c|c|}
\hline PERMAN & b & $\begin{array}{l}\text { personne ayant la permanence au moment } \\
\text { de la création ; }\end{array}$ \\
\hline ANNEES & $\mathbf{v}$ & $\begin{array}{l}\text { nombre d'années avec cette université } \\
\text { au moment de la création; }\end{array}$ \\
\hline QUITUNI? & $\mathrm{b}$ & $\begin{array}{l}\text { entrepreneur prévoyait de quitter l'université } \\
\text { dès la création de l'entreprise ou peu après ; }\end{array}$ \\
\hline TECHLIEE & $\mathrm{b}$ & $\begin{array}{l}\text { technologie utilisée par l'entreprise directement reliée } \\
\text { au travail universitaire de l'entrepreneur ; }\end{array}$ \\
\hline BREVUNI & b & $\begin{array}{l}\text { c'est l'université qui s'est chargée de faire } \\
\text { la demande de brevet ; }\end{array}$ \\
\hline FONDAT & $\mathbf{v}$ & nombre de fondateurs de l'entreprise ; \\
\hline GOVTD & $\%$ & $\begin{array}{l}\text { pourcentage des ventes de première année } \\
\text { faites au gouvernement; }\end{array}$ \\
\hline STYLED & & $\begin{array}{l}\text { style de gestion au moment du démarrage, } \\
\text { selon le fondateur (échelle continue, autoritaire }=0 \text {, } \\
\text { participatoire }=10 \text { ); }\end{array}$ \\
\hline OBJECTIF1 & o & $\begin{array}{l}\text { objectif au démarrage : devenir très grand } \\
\text { le plus vite possible; }\end{array}$ \\
\hline OBJECTIF2 & o & $\begin{array}{l}\text { objectif au démarrage : devenir le meilleur } \\
\text { pour la technologie; }\end{array}$ \\
\hline OBJECTIF3 & 0 & $\begin{array}{l}\text { objectif au démarrage : servir de nombreux marchés } \\
\text { avec des produits d'usage général ; }\end{array}$ \\
\hline OBJECTIF4 & $\mathbf{0}$ & $\begin{array}{l}\text { objectif au démarrage : être une seconde source } \\
\text { dans un marché industriel ; }\end{array}$ \\
\hline OBJECTIF5 & o & $\begin{array}{l}\text { objectif au démarrage : être le meilleur } \\
\text { dans un marché spécialisé; }\end{array}$ \\
\hline PLANAFF & b & plan d'affaire préparé au moment du démarrage ; \\
\hline PLANMRK & b & $\begin{array}{l}\text { plan marketing préparé au moment du démarrage } \\
\text { de l'entreprise ; }\end{array}$ \\
\hline ORIENTD & $\mathbf{v}$ & $\begin{array}{l}\text { orientation technologie }(=0) \text {, ou orientation marché }(=10) \\
\text { au moment du démarrage, échelle continue, estimé } \\
\text { par le fondateur; }\end{array}$ \\
\hline ENGAGEM & b & $\begin{array}{l}\text { au moment du démarrage, l'entrepreneur prévoyait } \\
\text { s'engager à long terme dans son entreprise ; }\end{array}$ \\
\hline XGESTION & $\mathbf{0}$ & $\begin{array}{l}\text { niveau de l'expérience de l'équipe de fondateurs } \\
\text { en gestion; }\end{array}$ \\
\hline XFINANCE & $\mathbf{0}$ & $\begin{array}{l}\text { niveau de l'expérience de l'équipe de fondateurs } \\
\text { en finance; }\end{array}$ \\
\hline XMARKET & 0 & $\begin{array}{l}\text { niveau de l'expérience de l'équipe de fondateurs } \\
\text { en marketing; }\end{array}$ \\
\hline XR\&D & 0 & $\begin{array}{l}\text { niveau de l'expérience de l'équipe de fondateurs } \\
\text { en } R \text { \& D; }\end{array}$ \\
\hline XVTEGOV & 0 & $\begin{array}{l}\text { niveau de l'expérience de l'équipe de fondateurs } \\
\text { en contrats avec le gouvernement; }\end{array}$ \\
\hline
\end{tabular}




\begin{tabular}{|c|c|c|}
\hline XDEMA & b & $\begin{array}{l}\text { expérience passée en démarrage d'entreprise } \\
\text { dans l'équipe de fondateurs; }\end{array}$ \\
\hline CAPDEP & $\mathbf{v}$ & capital de départ en dollars constants de 1986 ; \\
\hline ANEXPIND & $\mathbf{v}$ & $\begin{array}{l}\text { années d'expérience industrielle à temps plein } \\
\text { avant la création de l'entreprise ; }\end{array}$ \\
\hline PUBLI1 & $\mathbf{v}$ & $\begin{array}{l}\text { nombre de publications avec comité de lecture } \\
\text { et de présentations à des conférences, années } 2 \text { et } 3 \\
\text { avant la création (selon la déclaration de l'entrepreneur); }\end{array}$ \\
\hline FINRECH1 & $\mathbf{v}$ & $\begin{array}{l}\text { fonds de recherche reçus, années } 2 \text { et } 3 \text { avant la création } \\
\text { (fournie par l'entrepreneur); }\end{array}$ \\
\hline UNIVCAP & $\%$ & $\begin{array}{l}\text { capital initial venant de l'université (prêts, bourses, } \\
\text { capital); }\end{array}$ \\
\hline FONDCAP & $\%$ & capital initial venant du fondateur ou de ses partenaires ; \\
\hline FONDCTRD & $\%$ & $\begin{array}{l}\text { contrôle entre les mains de l'entrepreneur au moment } \\
\text { du démarrage ; }\end{array}$ \\
\hline GESTPROF & b & $\begin{array}{l}\text { gestionnaire professionnel passant plus de temps } \\
\text { que les fondateurs dans l'entreprise au moment } \\
\text { de sa création; }\end{array}$ \\
\hline TEMPSD & $\%$ & $\begin{array}{l}\text { temps passé par le fondateurs sur les affaires } \\
\text { de l'entreprise au moment de sa création; }\end{array}$ \\
\hline RETARD & $\mathbf{v}$ & $\begin{array}{l}\text { délai entre la création de l'entreprise } \\
\text { et ses premiers revenus (années); }\end{array}$ \\
\hline AUTFOND & $\%$ & fondateurs ne venant pas de l'université. \\
\hline
\end{tabular}

\section{Développement de l'entreprise et actlvités de l'entrepreneur ("Indicateurs de performance ")}

\begin{tabular}{|c|c|c|}
\hline CSi & $\mathbf{v}$ & $\begin{array}{l}\text { montant des ventes, année } i, i=1 \text { à } 5 \text {, en dollars constants } \\
\text { de } 1986 \text {; }\end{array}$ \\
\hline PUBLI2 & $\mathbf{v}$ & $\begin{array}{l}\text { nombre de publications avec comité de lecture } \\
\text { et de présentations à des conférences, années } 2 \text { et } 3 \\
\text { après la création (tel que l'a déclaré l'entrepreneur); }\end{array}$ \\
\hline FINRECH2 & $\mathbf{v}$ & $\begin{array}{l}\text { fonds de recherche reçus, années } 2 \text { et } 3 \text { après la création } \\
\text { (tel que l'a déclaré l'entrepreneur); }\end{array}$ \\
\hline FONDCTRM & $\%$ & $\begin{array}{l}\text { niveau de contrôle encore entre les mains } \\
\text { du principal fondateur; }\end{array}$ \\
\hline RATIODCAP & $\%$ & ratio, dettes à long terme sur avoir total, maintenant ; \\
\hline CAPRISQ & $\%$ & $\begin{array}{l}\text { niveau de contrôle présentement entre les mains } \\
\text { d'investisseurs risque ; }\end{array}$ \\
\hline AVECUNI & b & fondateur encore employé de l'université ; \\
\hline TEMPSM & $\%$ & $\begin{array}{l}\text { pourcentage du temps actuellement passé sur les affaires } \\
\text { de l'entreprise ; }\end{array}$ \\
\hline COBIEN & b & $\begin{array}{l}\text { estimation subjective par l'entrepreneur du bon état } \\
\text { de marche de l'entreprise. }\end{array}$ \\
\hline
\end{tabular}

